\title{
Species Distribution and Isolation Frequency of Nontuberculous Mycobacteria, Uruguay
}

\author{
Gonzalo Greif, Cecilia Coitinho, Jakko van Ingen, Carlos Robello
}

Nontuberculous mycobacteria (NTM) increasingly are recognized as opportunistic pathogens of humans. NTM species distribution is well documented in Europe and North America, but data from other regions are scarce. We assessed NTM isolation frequency and species distribution in Uruguay during 2006-2018.

$\mathrm{N}^{\mathrm{s}}$ Tontuberculous mycobacteria (NTM) are comprised of $>150$ species (1) and are increasingly recognized as opportunistic pathogens (2). In addition, frequency of disease-causing NTM isolation has been rising in many settings $(3,4)$. The exact epidemiology of NTM pulmonary disease, the most common manifestation in adults (3), has been difficult to determine because reporting is not mandatory in most countries and identification of true disease is often difficult (5).

The distribution of NTM species isolated from human clinical samples varies greatly by region (6). In South America, data on NTM isolation frequencies are available for São Paulo and Rio de Janeiro, Brazil (7-9) and Buenos Aires, Argentina (6). Because clinical and laboratory observations suggested an emergence of NTM isolates, we conducted a retrospective study of isolation frequency of NTM in Uruguay.

\section{The Study}

We conducted a retrospective study of data on all NTM isolates identified at the Comisión Honoraria de Lucha Anti-Tuberculosis (Montevideo, Uruguay), the national tuberculosis reference laboratory, during January 2006-December 2018. The laboratory

Author affiliations: Institut Pasteur Montevideo, Montevideo, Uruguay (G. Greif, C. Robello); Comisión Honoraria de Lucha Anti-Tuberculosa y Enfermedades Prevalentes, Montevideo (C. Coitinho); Radboud University Medical Center, Nijmegen, the Netherlands (J. van Ingen); Universidad de la República, Montevideo (C. Robello)

Article DOI: https://doi.org/10.3201/eid2605.191631 receives samples from all suspected tuberculosis (TB) cases across the country and performs universal acid-fast bacillus smear testing and solid culture in Löwenstein-Jensen and Ogawa egg-based media as a part of routine diagnostic testing. We abstracted basic patient demographic information and NTM identification results from the laboratory databases.

Phenotypic characterization of isolated NTM was performed by using biochemical methods, and complementary genotyping identification was introduced in 2012 by using GenoType Mycobacterium CM (Hain Lifescience GmbH, https:/ / www.hain-life science.de). In 2016, the laboratory included GenoType Mycobacterium AS (Hain Lifescience $\mathrm{GmbH}$ ) in its pipeline and retrospectively identified previously unidentified isolates.

For comparison, we also collected data on the number of culture-positive TB cases during the study period from the laboratory databases. We used official population information from the National Institute of Statistics Uruguay (Instituto Nacional de Estadística, http:/ /www.ine.gub.uy) to calculate NTM incidence (Appendix 1, https://wwwnc.cdc.gov/ EID/article/26/5/19-1631-App1.xlsx).

During 2006-2018, a total of 255 NTM isolates were collected from pulmonary and extrapulmonary samples from 204 patients, 143 male and 61 female, in Uruguay (Table 1). Most $(147 / 255 ; 57.6 \%)$ isolates identified were members of the Mycobacterium avium complex (MAC), which includes $M$. intracellulare and M. avium; 21 (8.2\%) were M. kansasii; 15 were M. gordonae (5.9\%); and 12 were M. peregrinum (4.7\%) (Table $2)$. We observed an increase in NTM isolation frequency and an increase in TB cases during 2011-2018 (Figure 1, panel A). We also calculated an age-adjusted isolation frequency to determine population aging effect (Figure 1, panel B).

Among 204 cases, 23 patients had >1 NTM-positive isolate 6 months apart, and 13 had positive cultures for $>2$ years. Patients $>50$ years of age (mean 
Table 1. Specimen sources for isolation of nontuberculous mycobacteria, Uruguay*

\begin{tabular}{lc}
\hline Specimen source & No. $(\%)$ \\
\hline Pulmonary & $170(66.6)$ \\
Sputum & $18(7.0)$ \\
Bronchoalveolar lavage & $8(3.1)$ \\
Bronchoalveolar secretions $†$ & $6(2.3)$ \\
Lung biopsy & $3(1.2)$ \\
Pleural fluid & $2(0.8)$ \\
Ear, nose, throat aspirate & $3(1.2)$ \\
Puncture fluid & $210(82.3)$ \\
Total & \\
\hline Extrapulmonary & $15(5.9)$ \\
Blood culture & $9(3.5)$ \\
Myelocyte culture & $4(1.6)$ \\
Skin or soft tissue abscess & $1(0.4)$ \\
Urine & $2(0.8)$ \\
Feces & $1(0.4)$ \\
Gastric lavage & $3(1.2)$ \\
Ganglion biopsy & $3(1.2)$ \\
Ascites fluid & $38(14.9)$ \\
Total & $3(1.2)$ \\
\hline Multiple isolation sources $\ddagger$ & $4(1.6)$ \\
Missing data & \\
*The data include isolates from the same patient over time. \\
†ncludes tracheal aspirations. \\
†lncludes pulmonary and nonpulmonary samples of the same patient. \\
\hline
\end{tabular}

52 years of age) were more likely to have prolonged culture-positivity, a statistically significant difference from patients with a single positive culture (mean 42.6 years of age; $p=0.0099)$. Most $(7 / 13 ; 53.8 \%)$ prolonged infections were caused by $M$. intracellulare, and 4 prolonged cases remained NTM-positive for $\leq 6$ years, including 2 infections with $M$. intracellulare and 1 each with $M$. kansasii and $M$. heckeshornense. Most $(10 / 13 ; 76.9 \%)$ prolonged cases were detected in pulmonary isolates.
Only $4(1.9 \%)$ cases showed disseminated infections in isolates obtained from hemoculture, bone marrow culture, ganglion biopsy, feces, urine, or sputum of the same patient. Among the 4 disseminated infections, $M$. avium was isolated from 2 cases, $M$. genavense from 1 case, and $M$. intracellulare from 1 case.

The incidence of NTM in Uruguay increased from 0.33 cases $/ 100,000$ inhabitants in 2006 to 1.57 cases $/ 100,000$ inhabitants to 2018 (Figure 1, panel A). In 2018, the incidence of NTM was $2.73 / 100,000$ inhabitants in the north, which has only $16.72 \%$ of the total population of the country. In the south, where $83.28 \%$ of the population lives, the incidence was much lower, 1.34 cases/100,000 inhabitants. Culturepositive TB cases showed a reverse tendency with statistically significant differences. The incidence rate for TB was higher in the south, 30.01 cases $/ 100,000$ inhabitants, and lower in the north, 21.50 cases/100,000 inhabitants (Figure 2).

During the period studied, we observed a 4 -fold increase in the NTM isolation rate (Appendix 1). Implementation of new detection techniques during this timeframe could account for part of the increase (10). However, we noted a $>2$-fold increase (2.49) in number of isolates recorded during 20172018, when no laboratory protocol changes were introduced, suggesting the NTM incidence is rising in Uruguay (Appendix 1).

Rivero-Lezcano et al. (10) suggested changes in the pathogen and an aging population could explain the rising incidence of NTM isolation and

\begin{tabular}{|c|c|c|c|c|c|c|c|c|c|c|c|c|c|c|c|}
\hline Species & 2006 & 2007 & 2008 & 2009 & 2010 & 2011 & 2012 & 2013 & 2014 & 2015 & 2016 & 2017 & 2018 & Total & \% Total \\
\hline Mycobacterium intracellulare & 6 & 5 & 2 & 2 & 4 & 3 & 6 & 3 & 6 & 13 & 9 & 10 & 17 & 86 & 33.7 \\
\hline M. avium & 4 & 0 & 1 & 1 & 7 & 2 & 3 & 2 & 5 & 6 & 3 & 6 & 21 & 61 & 23.9 \\
\hline M. kansasii & 0 & 0 & 0 & 1 & 2 & 3 & 2 & 2 & 5 & 1 & 2 & 2 & 1 & 21 & 8.2 \\
\hline M. gordonae & 2 & 1 & 0 & 0 & 0 & 0 & 0 & 2 & 4 & 2 & 0 & 2 & 2 & 15 & 5.9 \\
\hline M. peregrinum & 0 & 0 & 0 & 0 & 1 & 1 & 1 & 0 & 1 & 1 & 3 & 2 & 2 & 12 & 4.7 \\
\hline M. chelonae & 0 & 0 & 0 & 0 & 0 & 0 & 1 & 1 & 1 & 1 & 2 & 0 & 2 & 8 & 3.1 \\
\hline M. fortuitum & 0 & 0 & 0 & 0 & 0 & 0 & 0 & 2 & 1 & 0 & 1 & 2 & 2 & 8 & 3.1 \\
\hline $\begin{array}{l}\text { NTM, no species } \\
\text { determinant }\end{array}$ & 0 & 0 & 0 & 0 & 0 & 0 & 0 & 0 & 0 & 1 & 3 & 1 & 0 & 5 & 2.0 \\
\hline M. genavense & 0 & 0 & 0 & 0 & 0 & 0 & 0 & 0 & 0 & 3 & 1 & 0 & 0 & 4 & 1.6 \\
\hline M. avium MTBC $†$ & 0 & 0 & 0 & 0 & 0 & 1 & 0 & 2 & 0 & 0 & 0 & 0 & 0 & 3 & 1.2 \\
\hline M. intracellulare MTBC $\dagger$ & 0 & 0 & 0 & 0 & 1 & 0 & 0 & 0 & 2 & 0 & 0 & 0 & 0 & 3 & 1.2 \\
\hline M. Ientiflavum & 0 & 0 & 0 & 0 & 0 & 0 & 0 & 0 & 0 & 1 & 0 & 2 & 1 & 4 & 1.6 \\
\hline M. scrofulaceum & 0 & 0 & 0 & 0 & 0 & 2 & 0 & 0 & 0 & 0 & 1 & 0 & 1 & 4 & 1.6 \\
\hline M. simiae & 0 & 1 & 0 & 0 & 0 & 0 & 0 & 0 & 0 & 0 & 2 & 0 & 0 & 3 & 1.2 \\
\hline M. abscessus & 0 & 0 & 0 & 1 & 0 & 0 & 1 & 0 & 0 & 0 & 0 & 0 & 1 & 3 & 1.2 \\
\hline M. heckeshornense & 0 & 0 & 0 & 0 & 0 & 0 & 0 & 1 & 1 & 0 & 0 & 0 & 0 & 2 & 0.8 \\
\hline M. xenopi & 0 & 0 & 1 & 0 & 0 & 0 & 0 & 1 & 0 & 0 & 0 & 0 & 0 & 2 & 0.8 \\
\hline Other species $\ddagger$ & 0 & 0 & 0 & 0 & 0 & 0 & 0 & 3 & 0 & 4 & 0 & 1 & 3 & 11 & 4.3 \\
\hline Total & 12 & 7 & 4 & 5 & 15 & 12 & 14 & 19 & 26 & 33 & 27 & 28 & 53 & 255 & 100.0 \\
\hline
\end{tabular}

${ }^{*}$ MTBC, Mycobacterium tuberculosis complex; NTM, nontuberculous mycobacterium.

†Mixed infections.

fOther species included 1 isolate each of $M$. arupense, $M$. asiaticum, $M$. koreense, $M$. shimodei, $M$. interjectum, M. marinum and $M$. malmoense, and mixed infections of $M$. avium-M. intracellulare, M. gordonae-M. chelonae, M. intracellulare, $M$. fortuitum, $M$. intracellulare-MTBC. 


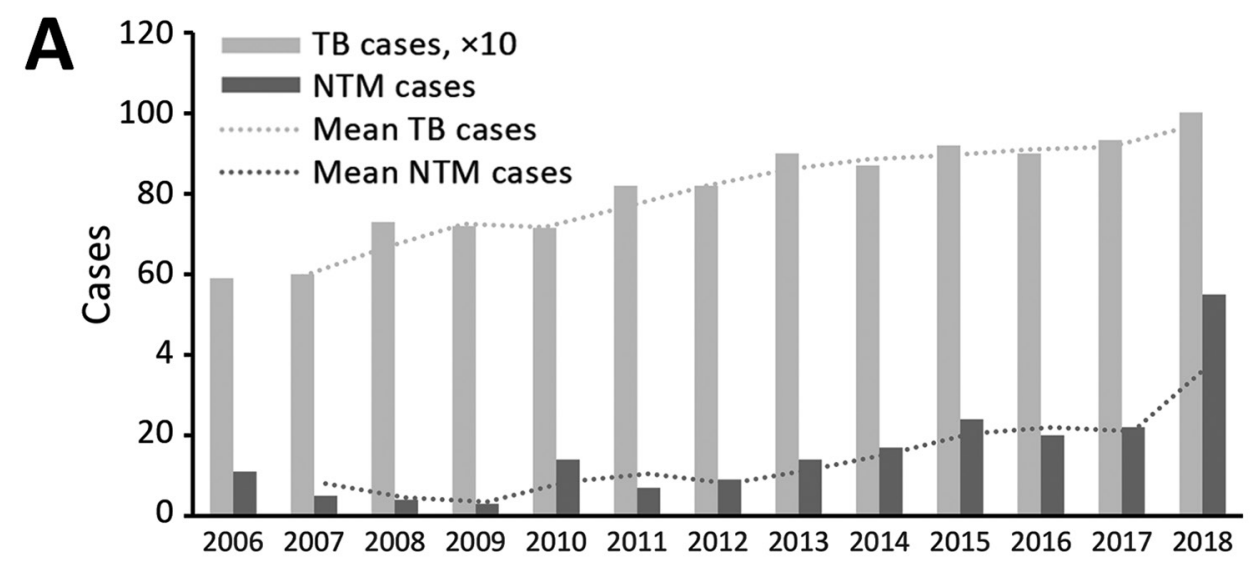

Figure 1. Number of cases of nontuberculous mycobacteria and tuberculosis, Uruguay, 2006-2018. A) NTM and TB cases by year. B) NTM incidence adjusted by age range and year. NTM, nontuberculous mycobacteria; TB, tuberculosis.

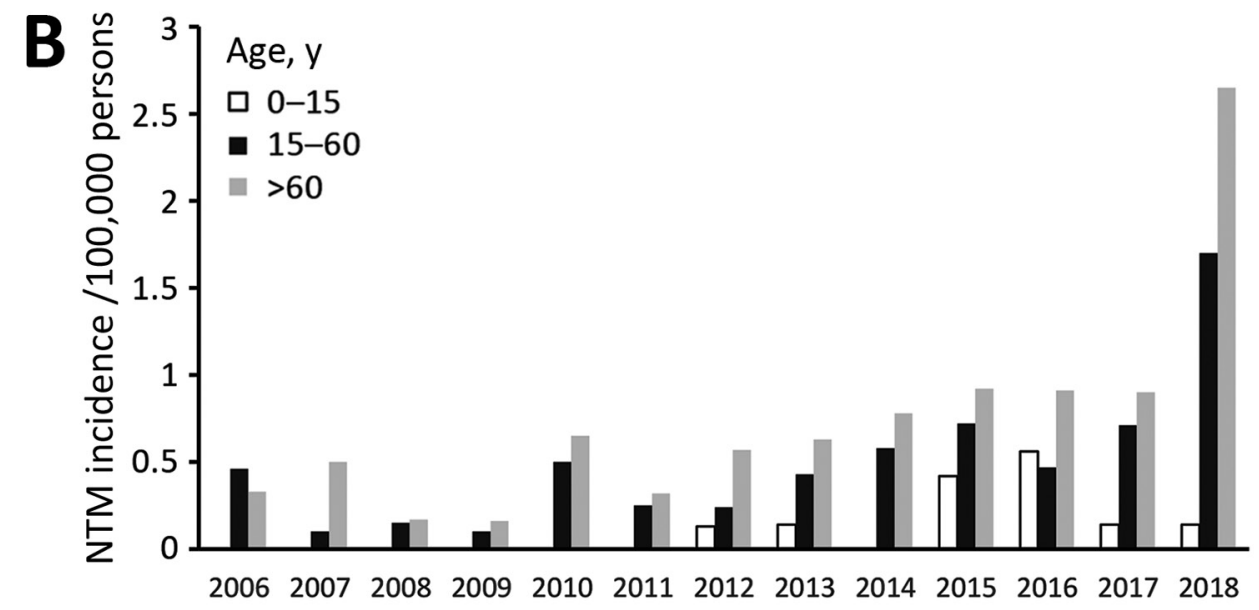

disease. From 2017 to 2018, we observed a higher increase in NTM incidence (2.94 cases/100,000 persons) among persons $>60$ years of age compared with persons $15-60$ years of age (2.39 cases/100,000 persons) (Figure 1, panel B). However, we noted that the age composition of the population remained stable over time (Appendix 1), suggesting other factors are driving increases in NTM isolation rates.

MAC is reported to be the most frequently isolated NTM species in all continents (3), but we observed a different species distribution than previously reported for South America. We saw a higher prevalence of $M$. intracellulare (33.7\% of isolates) than $M$. avium (23.9\% of isolates) (Table 2 ). We found species not previously reported in Latin America, including M. xenopi and M. arupense, and M. malmoense, which typically is found in northern Europe (11). We also found M. parakoreense, for which only 2 cases are reported in the literature, 1 case in 2013 isolated from a single clinical sample (12) and another in Africa (13). In addition, we noted 2 cases of $M$. heckeshornense, a species previously reported by our group (14).
We noted prolonged positivity in $6 \%(13 / 204)$ of NTM cases. MAC was responsible for most (69\%) prolonged culture positives and M. kansasii was responsible for $15 \%$ of prolonged positivity.

We believe the differences observed in NTM isolation rates between regions of Uruguay could be explained by climate factors. The higher temperatures and increased rainfall in the north could favor the incidence ambient pathogens (Appendix 2, https://wwwnc.cdc.gov/EID/article/26/5/191631-App2.pdf). We also cannot disregard differences associated with other factors, such as the age of the population.

Our study has several limitations. First, the public health system of Uruguay only registers $M$. tuberculosis; clinical data of NTM patients are difficult to obtain. In addition, the GenoType AS assay used to identify NTM does not detect all NTM species; including genomic sequencing-based identification methods would improve detection in the future. Identification of NTM species is crucial for determining appropriate clinical and antimicrobial treatment for pulmonary NTM, which are dependent on the characteristics of the species (8). 

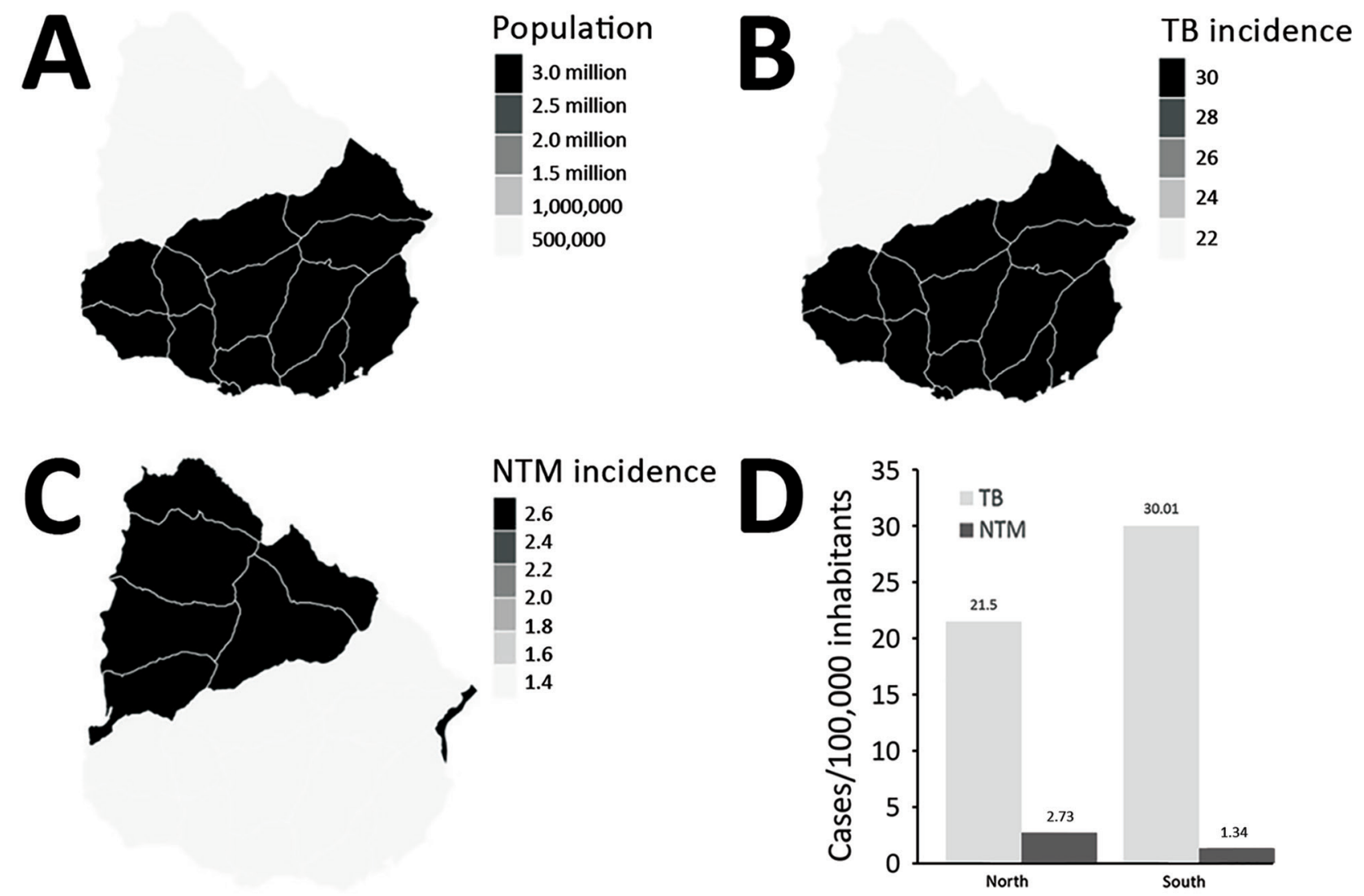

Figure 2. Distribution of cases of tuberculosis and nontuberculous mycobacteria by region and population density in 2018, Uruguay. A) Population density of north versus south regions of Uruguay. B) Incidence of TB cases in 2018. C) Incidence of NTM cases in 2018. D) TB and NTM incidence by region. Even though the population density in the north is much lower than that in the south, the north has a higher incidence of NTM than the south. NTM, nontuberculous mycobacteria; TB, tuberculosis.

\section{Conclusions}

Our findings indicate that rate of NTM have risen nearly 5-fold (4.79) in Uruguay, from 0.33 cases/100,000 inhabitants in 2006 to 1.57 cases/100,000 inhabitants in 2018. The species distribution largely aligns with existing data from elsewhere in South America, which demonstrates a predominance of MAC. Prolonged culture positivity and various isolation sites sources suggest that NTM disease is prevalent in Uruguay and warrants further studies to optimize diagnosis and treatment.

\section{Acknowledgments}

We thank Carlos Rivas for his support and efforts to preserve nontuberculous mycobacteria data at the Comisión Honoraria de Lucha Anti-Tuberculosis.

G.G. and C.R. are supported by the Sistema Nacional de Investigadores, Uruguay.

\section{About the Author}

Dr. Greif is a molecular biologist at the Institut Pasteur Montevideo, Montevideo, Uruguay. His research interests include developing molecular methods for diagnosing mycobacteria.

\section{References:}

1. Tortoli E. Microbiological features and clinical relevance of new species of the genus Mycobacterium. Clin Microbiol Rev. 2014;27:727-52. https://doi.org/10.1128/ CMR.00035-14

2. Griffith DE, Aksamit T, Brown-Elliott BA, Catanzaro A, Daley C, Gordin F, et al.; ATS Mycobacterial Diseases Subcommittee; American Thoracic Society; Infectious Disease Society of America. An official ATS/IDSA statement: diagnosis, treatment, and prevention of nontuberculous mycobacterial diseases. Am J Respir Crit Care Med. 2007;175:367-416. https://doi.org/10.1164/ rccm.200604-571ST

3. Zweijpfenning SMH, Ingen JV, Hoefsloot W. Geographic distribution of nontuberculous mycobacteria isolated from clinical specimens: a systematic review. 
Semin Respir Crit Care Med. 2018;39:336-42. https://doi.org/10.1055/s-0038-1660864

4. Schiff HF, Jones S, Achaiah A, Pereira A, Stait G, Green B. Clinical relevance of non-tuberculous mycobacteria isolated from respiratory specimens: seven year experience in a UK hospital. Sci Rep. 2019;9:1730. https:/ / doi.org/10.1038/ s41598-018-37350-8

5. Prevots DR, Loddenkemper R, Sotgiu G, Migliori GB. Nontuberculous mycobacterial pulmonary disease: an increasing burden with substantial costs. Eur Respir J. 2017;49:1700374. https://doi.org/10.1183/ 13993003.00374-2017

6. Hoefsloot W, van Ingen J, Andrejak C, Ängeby K, Bauriaud R, Bemer P, et al.; Nontuberculous Mycobacteria Network European Trials Group. The geographic diversity of nontuberculous mycobacteria isolated from pulmonary samples: an NTM-NET collaborative study. Eur Respir J. 2013;42:1604-13. https:/ / doi.org/10.1183/ 09031936.00149212

7. Bensi EP, Panunto PC, Ramos MC. Incidence of tuberculous and non-tuberculous mycobacteria, differentiated by multiplex PCR, in clinical specimens of a large general hospital. Clinics (São Paulo). 2013; 68:179-84. https:// doi.org/10.6061/clinics/ 2013(02)OA10

8. Marques LRM, Ferrazoli L, Chimara É. Pulmonary nontuberculous mycobacterial infections: presumptive diagnosis based on the international microbiological criteria adopted in the state of São Paulo, Brazil, 2011-2014 [in Portuguese]. J Bras Pneumol. 2019;45:e20180278. https:/ / doi.org/10.1590/1806-3713/e20180278

9. de Mello KGC, Mello FC, Borga L, Rolla V, Duarte RS, Sampaio EP, et al. Clinical and therapeutic features of pulmonary nontuberculous mycobacterial disease, Brazil, 1993-2011. Emerg Infect Dis. 2013;19:393-9.

10. Rivero-Lezcano OM, González-Cortés C, Mirsaeidi M. The unexplained increase of nontuberculous mycobacteriosis. Int J Mycobacteriol. 2019;8:1-6. https://doi.org/10.4103/ijmy.ijmy_18_19

11. Jankovic M, Samarzija M, Sabol I, Jakopovic M, Katalinic Jankovic V, Zmak L, et al. Geographical distribution and clinical relevance of non-tuberculous mycobacteria in Croatia. Int J Tuberc Lung Dis. 2013; 17:836-41. https://doi.org/10.5588/ijtld.12.0843

12. Kim BJ, Hong SH, Yu HK, Park YG, Jeong J, Lee SH, et al. Mycobacterium parakoreense sp. nov., a slowly growing non-chromogenic species related to Mycobacterium koreense, isolated from a human clinical specimen. Int J Syst Evol Microbiol. 2013;63:2301-8. https://doi.org/10.1099/ ijs.0.045070-0

13. Chin'ombe N, Muzividzi B, Munemo E, Nziramasanga P. Molecular identification of nontuberculous mycobacteria in humans in Zimbabwe using $16 \mathrm{~S}$ ribosequencing. Open Microbiol J. 2016;10:113-23. https://doi.org/10.2174/ 1874285801610010113

14. Coitinho C, Greif G, van Ingen J, Laserra P, Robello C, Rivas C. First case of Mycobacterium heckeshornense cavitary lung disease in the Latin America and Caribbean region. New Microbes New Infect. 2015;9:63-5. https:/ / doi.org/ 10.1016/j.nmni.2015.12.003

Address for correspondence: Gonzalo Greif, Laboratorio de Interacción Hospedero-Patógeno, Unidad de Biología Molecular, Institut Pasteur Montevideo, Mataojo 2020, Montevideo, Uruguay; email: gonzalo.greif@pasteur.edu.uy

\section{EID Podcast: Epidemiology of Human Plague in the United States, 1900-2012}

Plague is a globally distributed, zoonotic disease caused by the bacterium Yersinia pestis. In the late 1890s, rat-infested steamships introduced the disease into the continental United States. The first documented autochthonous human infection occurred in the Chinatown section of San Francisco, California, in March of 1900. Cases were soon reported in other port cities, including New Orleans, Galveston, Seattle, and Los Angeles. Along the Pacific Coast, infection spread from urban rats to native rodent species, and by the 1950s, Y. pestis had spread eastward to reach western portions of the Dakotas, Nebraska, Kansas, Oklahoma, and Texas. This distribution has remained static for more than 60 years, presumably the result of climatic and ecologic factors that limit further spread. Although poorly defined, these factors may be related to the ecology of vector species rather than that of rodent hosts.

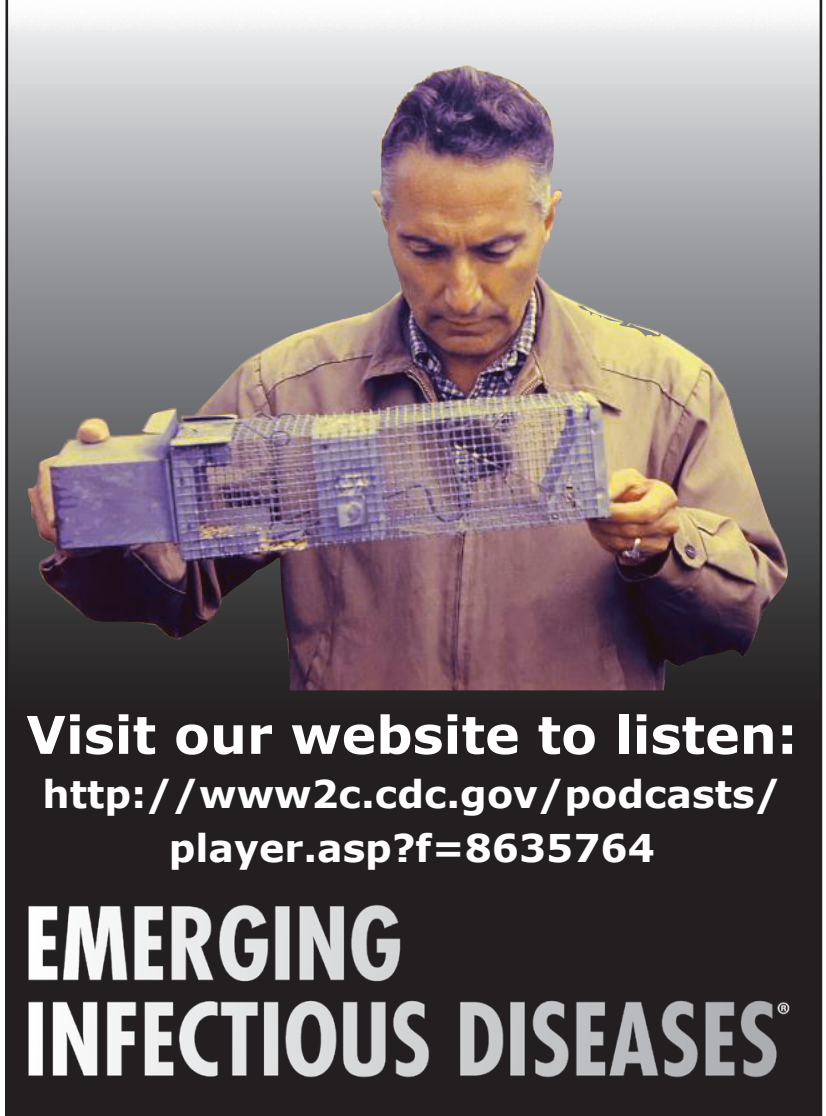

